\title{
Evaluation of the effect of an educational intervention on knowledge and adherence to HIV guidelines among frontline health workers in Alex-Ekwueme Federal University Teaching Hospital Abakaliki, Nigeria
}

\author{
Ifeyinwa Chizoba Akamike ${ }^{1,2}$, Ijeoma Nkem Okedo-Alex ${ }^{1,2}$, Chigozie Jesse Uneke ${ }^{1}$, Henry \\ Chukwuemeka Uro-Chukwu' ${ }^{1}$, Onyedikachi Echefu Chukwu' ${ }^{1}$, Ngozi Immaculata \\ $\mathrm{Ugwu}^{1,3}$, Urudinachi Nnenne Agbo ${ }^{1,2}$, Abel Ebeh Ezeoha ${ }^{1}$
}

\begin{abstract}
1. African Institute for Health Policy and Health Systems, Ebonyi State University, Abakaliki.
2. Department of Community Medicine, Alex-Ekwueme Federal University Teaching Hospital Abakaliki Ebonyi State Nigeria.

3. Department of Haematology, Faculty of Clinical Medicine, Ebonyi State University, Abakaliki.
\end{abstract}

\begin{abstract}
Author details:
Chigozie Jesse Uneke: unekecj@yahoo.com, +2348038928597; Ijeoma Nkem Okedo-Alex: ijeomaninadr@gmail.com, +2348064927291; Henry Chukwuemeka Uro-Chukwu: hurochu@gmail.com, +2348170616546; Onyedikachi Echefu Chukwu: onyedikachichukwu@gmail.com,+2348036263206;

Ngozi Immaculata Ugwu: ngoziugwu5@gmail.com, +2348061177100; Urudinachi Nnenne Agbo: urudinachiagbo@gmail.com, +2348037452026; Abel Ezeoha: aezeoha@gmail.com, +2347033665383
\end{abstract}

\begin{abstract}
Background: The aim of this study was to appraise the implementation of the National HIV guidelines and determine the effect of an educational intervention on health worker knowledge and practice of the guidelines.

Methods: A before and after study design without control was carried out using a self-administered questionnaire and key informant interviews. Data was also collected from client record cards. An educational intervention was carried out using pamphlets containing summarized information on the guideline. Data analysis was carried out using IBM-SPSS version 20. Result: Results showed that $54.5 \%$ of the respondents were males and $76 \%$ were medical doctors. Baseline knowledge level of respondents was high with $97 \%$ of respondents having good knowledge with a mean score of 3.9. This increased to 4.1 out of 5 post-intervention. All respondents had good practice of the guidelines before and after intervention with a mean score of 4.5 out of 5 . Client records also showed good practice. Barriers to guideline implementation include: poor knowledge, inadequate training, guideline unavailability, poor functioning of the laboratory equipment, poor funding.

Conclusion: HIV guidelines are being implemented in the clinic to a large extent; however, trainings, funding and provision of the guideline in the clinics are recommended.

Keywords: HIV, guideline implementation, health worker.

DOI: https://dx.doi.org/10.4314/ahs.v20i3.10

Cite as: Akamike IC, Okedo-Alex IN, Uneke CJ, Uro-Chukwn HC, Chukwn OE, Ugwn NI, et al. Evaluation of the effect of an educational intervention on knowledge and adherence to HIV guidelines among frontline health workers in Alex-Ekwueme Federal University Teaching Hospital Abakaliki, Nigeria. Afri Health Sci. 2020;20(3): 1080-1089. https:/ / dx.doi.org/10.4314/ahs.v20i3.10
\end{abstract}

\section{Corresponding author:}

Ifeyinwa Chizoba Akamike

Department of Community Medicine,

Alex-Ekwueme Federal University Teaching

Hospital Abakaliki Ebonyi State Nigeria;

African Institute for Health Policy and Health

Systems, Ebonyi State University, Abakaliki

Phone: +2347068045271

Email: ifeyakamike@gmail.com;

ifeychima@yahoo.com

\section{Introduction}

Over the years, medical advancement has helped to increase the life expectancy among people living with HIV (Human immunodeficiency virus). Despite these advances, some segments of the population have not achieved these increases in life expectancy and usually progress to the end stages of the disease. ${ }^{1}$ In order to achieve better health outcomes and for provision of antiretroviral therapy (ART) to be effective, it is necessary to provide quality HIV/AIDS care. ${ }^{2}$ Use of guidelines have been shown to improve the quality of care by re- 
ducing disparities in practice and adherence to standards of good care outcomes. ${ }^{3,4}$ Effective implementation of clinical guidelines can lead to improvement in clinical outcomes, reduced hospital stay, and reduced frequency of monitoring and cost. ${ }^{4}$

In Nigeria, the National Guidelines on the use of ARV (anti-retroviral) drugs was first developed in 2003. This served as the template for the development of National Guidelines for HIV and AIDS Treatment and Care in Adults and Children, and National Guidelines for Prevention of Mother-to-Child Transmission of HIV in 2005. The 2005 edition of the guideline on treatment and care was later developed but was considered inadequate for management of HIV in children. This stimulated the creation of separate documents for children and adults in 2007 which were later updated in 2010 as a result of new developments in the management of HIV. The recommendations from the World Health Organization (WHO) consolidated guidelines in 2013 prompted the review and harmonization of the three guidelines into a set of consolidated guidelines based on current scientific evidence and practices. ${ }^{5}$

These guidelines provide guidance on the diagnosis of HIV infection, the care of people living with HIV and the use of ARV drugs for treating and preventing HIV infection. ${ }^{6}$

In the ART clinic of Alex-Ekwueme Federal University Teaching Hospital Abakaliki, the 2016 version of the Nigerian national guideline is being used. It provides the guidance that health workers need to deliver comprehensive and quality HIV care. The key recommendations are: initiation of ART in all those who test positive for HIV including children, adults, adolescents, pregnant and breastfeeding mothers, regardless of clinical or immunological status. ${ }^{7}$ Health workers in general have a role to play in improving treatment of HIV patients. Health worker's compliance to the HIV management guideline is of great importance in maintaining high quality in the management of HIV. Previous studies revealed varying levels of health worker knowledge of HIV guidelines. ${ }^{8-12}$ Some studies have shown that adherence of health workers to HIV/AIDS diagnostic and treatment guidelines is generally poor. ${ }^{2,13}$

The purpose of this study was to appraise the implementation of the HIV management guideline in the adult ART clinic of Alex Ekwueme Federal University Teaching Hospital Abakaliki and to determine the effect of an educational intervention on the implementation of the guideline by health workers.

\begin{tabular}{|c|c|}
\hline \multicolumn{2}{|c|}{ Some key extracts from the 2016 HIV guidelines } \\
\hline $\begin{array}{l}\text { For HIV positive adults and } \\
\text { adolescents }\end{array}$ & $\begin{array}{l}\text { 1. Initiate ART in all adults and adolescents with a } \\
\text { diagnosis of HIV regardless of clinical stage of disease } \\
\text { and CD4+ cell count. } \\
\text { 2. Dolutegravir (DTG) and Efavirenz (EFV) } 400 \mathrm{mg} \text { are } \\
\text { new alternatives in first line ART regimens. } \\
\text { 3. The guideline recommends early initiation } \\
\text { of therapyas soon as possible preferably within two } \\
\text { weeks of diagnosis of HIV infection }\end{array}$ \\
\hline Adherence & $\begin{array}{l}\text { 1. Continuous adherence counselling is essential in ART } \\
\text { and should be accessible to every patient on ART. } \\
\text { This should include adherence assessments and } \\
\text { documentation at every visit }\end{array}$ \\
\hline $\begin{array}{l}\text { Preventing Opportunistic } \\
\text { Infections }\end{array}$ & $\begin{array}{l}\text { 1. Due to high prevalence of malaria and severe bacterial } \\
\text { infections in Nigeria,co-trimoxazole prophylaxis } \\
\text { should be initiated regardless of CD4+ cell count or } \\
\text { WHO stage. } \\
\text { 2. Adults living wth HIV should be screened using a } \\
\text { clinical algorithm; those who do not report any one of } \\
\text { the symptoms of current cough, fever, weight loss, or } \\
\text { night sweat are unlikely to have active TB and should } \\
\text { be offered Isoniazid Preventive Therapy }\end{array}$ \\
\hline
\end{tabular}




\section{Methods}

The study was carried out in the adult antiretroviral therapy clinic of Alex-Ekwueme Federal University Teaching Hospital Abakaliki (AE-FUTHA) in 2017. The Hospital has over 4000 staff, comprising about 200 consultants in various specialties, 602 beds, ward complex distributed in various departments, about 500 doctors undergoing residency training and a capacity for training over 250 house officers every year. AE-FUTHA serves as the largest comprehensive HIV/AIDs care centre in the state. A total of about 1500 adult clients were on treatment in the facility as at the time of this study. The adult clinic runs weekly from Monday to Thursday. A total of about 40 health workers work in the clinic.

\section{Study design}

The study was an intervention study without control or randomization and was conducted in three phases: pre-intervention, intervention and post intervention surveys.

The study population comprised of health care workers who render services at the HIV clinic. Healthcare workers who are not directly involved in patient care and who were on leave at the time of the study were excluded from the study. The study was a total population study. All the eligible health workers working in the clinic were selected for the study. A total of 33 health workers were surveyed. A pre-tested semi-structured, self-administered questionnaire was used to collect information from respondents. In addition to the questionnaire, a checklist was used to collect information from 100 patient treatment cards to verify the reported practice of health workers. Parameters that were assessed using the checklist include WHO staging, screening for tuberculosis, prescription of cotrimoxazole, assessment of adherence, baseline investigation and pre-treatment adherence counseling.

Key informant interviews were conducted to gather more information about guidelines implementation and major factors/challenges limiting implementation.
There were four respondents for the key informant interview some of whom were in leadership positions in the clinic at the time of the study.

\section{Data Collection Methods \\ Pre-intervention survey}

Pre-intervention data was collected using a pretested, structured self- administered questionnaire to collect information from health workers including health worker's demographic characteristics, their knowledge, practice/adherence to guidelines for HIV and factors affecting implementation of the guidelines. Data was also collected from client's clinical records using a checklist. Respondents were informed that whatever information they give will not be made known to any other person.

\section{Intervention}

The intervention for this study included provision of information to health workers on the guidelines. This was done through provision of leaflets containing information on the National HIV management guidelines. The pamphlet was prepared using the National Guidelines. Relevant sections of the guidelines were summarized highlighting important key points particularly the new aspects of the guidelines. The leaflet was distributed to the health workers and was also placed at the various consulting rooms to make for easy access to the summarized version. After distribution of the pamphlet, the health workers were reminded and encouraged to read it as many times as they could. The intervention lasted for a period of one month. (Figure 1)

\section{Post Intervention Survey}

Three months after the intervention, the same questionnaire that was used for pre-intervention data collection was administered to the same respondents that were surveyed pre-intervention. The same checklist that was used pre-intervention was also used for data abstraction from the client records. The purpose was to determine the effect of the intervention on implementation of the HIV management guidelines. 


\section{- One Month}

\section{- One month}

Period

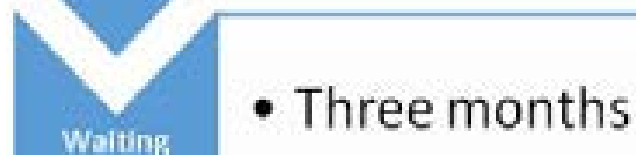

period

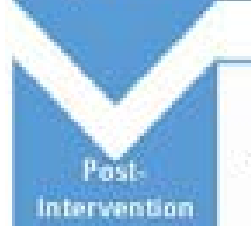

- One month

Survey

Figure 1: Study Timeline Diagram

\section{Data Management}

\section{Measurement of Variables}

The independent variables included: socio-demographic characteristics of health workers such as age, gender, marital status, professional level, and duration of service in ART clinic.These variables were measured using the structured questionnaire. Frequencies and proportions were calculated for categorical variables while the means and standard deviations were calculated for numeric/quantitative variables.

The dependent variables included:

Knowledge: There were eight questions that assessed the knowledge of healthcare providers on HIV guidelines. Each question has five options on a rating scale of 1-5 points, where 1point=strongly disagree; 2 points $=$ disagree; 3 points $=$ indifferent 4 points $=$ agree and 5 points=strongly agree. Values ranging from 1.002.99 points were considered poor knowledge whereas values ranging from 3.00-5.00 points considered high. The Pre-intervention means were compared to the Post- intervention means.

\section{Extent of implementation of HIV treatment}

Guidelines: Eight questions were used to assess the extent to which the guidelines are being implemented. This was also based on a 5 point likert scale. Each question had five options on a rating scale of $1-5$ points, where 1 point $=$ Never; 2 points $=$ rarely; 3 points $=$ sometimes; 4 points $=$ often times and 5 points $=$ always. Values ranging from 1.00-2.99 points were considered poor practice whereas values ranging from 3.00-5.00 points were considered good practice. The pre-intervention means were compared to the post- intervention means. 


\section{Statistical analysis}

Statistical Package for Social Sciences (IBM-SPSS) for Microsoft Window version 20 software was used for the data analysis. Frequency tables were used to present the descriptive statistics of the variables, and relevant means, standard deviations, and proportions were calculated. For the likert scale questions, analysis was based on mean neutral rating (MNR). Values ranging from 1.00-2.99 points were considered poor knowledge whereas values ranging from 3.00-5.00 points were considered high. The pre-intervention means were compared to the post- intervention means.

\section{Ethical considerations}

Ethical clearance for this study was obtained from Re- search and Ethics Committee of Ebonyi State University and Alex-Ekwueme Federal University Teaching Hospital Abakaliki, Ebonyi State of Nigeria. In order to ensure confidentiality, the data collection tools did not include self-identifying characteristics such as participant's name and contacts. Participants were informed of the purpose of the study and their roles and rights as participants. Their consent to participate was sought and documented using an informed consent form. Participants were also informed that participation in the research was voluntary.

\section{Result}

Table 1 shows that majority of the respondents were 34-43 years, females and were married.

Table 1: Socio-demographic characteristics of health workers in the adult HIV clinic

\begin{tabular}{ll}
\hline Variable & $\mathbf{N = 3 3}$ \\
\hline Age group & $\mathbf{n}(\mathbf{\%})$ \\
$24-33$ & $10(30.3)$ \\
$34-43$ & $18(54.6)$ \\
$44-54$ & $5(15.1)$ \\
Gender & \\
Male & $15(45.5)$ \\
Female & $18(54.5)$ \\
Marital status & \\
Single & $3(9.1)$ \\
Married & $26(78.7)$ \\
Widowed & $2(6.1)$ \\
Divorced & $2(6.1)$ \\
Professional Status & \\
Medical Doctors & $25(75.8)$ \\
Other health workers & $8(24.2)$ \\
Duration of work in HIV Clinic & \\
6months- $<$ lyear & $5(15.2)$ \\
1-2 years & $5(15.2)$ \\
3-5 years & $17(51.5)$ \\
$>5$ years & $6(18.1)$ \\
\hline
\end{tabular}


Table 2: Difference in Pre/Post mean scores for knowledge of HIV guidelines

\begin{tabular}{llll}
\hline Knowledge Item & Pre-Mean(SD) & $\begin{array}{l}\text { Post- } \\
\text { Mean(SD) }\end{array}$ & \%Mean Difference \\
\hline Knowledge level & $3.1 \pm 1.4$ & $3.1 \pm 1.4$ & $0 \%$ \\
$\begin{array}{l}\text { It is important to carry out } \\
\text { baseline investigations }\end{array}$ & $4.5 \pm 0.9$ & $4.6 \pm 1.1$ & $2.2 \%$ \\
$\begin{array}{l}\text { CD4 count should be done } \\
\text { every } 6 \text { months }\end{array}$ & $4.3 \pm 1.2$ & $4.8 \pm 0.8$ & $11.6 \%$ \\
$\begin{array}{l}\text { ART should be commenced } \\
\text { regardless of WHO staging or } \\
\text { CD4 count }\end{array}$ & $4.0 \pm 1.4$ & $3.8 \pm 1.6$ & $-5 \%$ \\
$\begin{array}{l}\text { WHO staging should be done } \\
\text { at every visit }\end{array}$ & $4.2 \pm 0.9$ & $4.7 \pm 0.5$ & $11.9 \%$ \\
$\begin{array}{l}\text { Screening for TB should be } \\
\text { done at every visit }\end{array}$ & $3.7 \pm 1.5$ & $4.2 \pm 1.2$ & $13.5 \%$ \\
$\begin{array}{l}\text { Monitoring of drug toxicity } \\
\text { should be done }\end{array}$ & $4.6 \pm 0.8$ & $4.8 \pm 0.6$ & $4.4 \%$ \\
$\begin{array}{l}\text { Knowledge of monitoring } \\
\text { toxicity }\end{array}$ & $2.7 \pm 1.2$ & $3.0 \pm 1.3$ & $11.1 \%$ \\
\hline
\end{tabular}

Table 2 shows that there was an increase in mean knowledge scores for majority of the knowledge items with knowledge about WHO staging having the highest increase $(11.9 \%)$. Others increased as follows: $2.2 \%$ for carrying out baseline investigations, $11.6 \%$ for CD4 at every 6 months, $13.5 \%$ for screening for TB at every visit, $4.4 \%$ monitoring of drug toxicity and $11.1 \%$ for knowledge of monitoring toxicity. There was however a decrease in the mean score for knowledge about commencement of ART

Table 3: Difference in Pre/Post mean scores for practice of the HIV guidelines

\begin{tabular}{|c|c|c|c|}
\hline Practice Item & Pre-Mean & $\begin{array}{l}\text { Post- } \\
\text { mean }\end{array}$ & $\%$ Mean Difference \\
\hline $\begin{array}{l}\text { Commencement of ART based } \\
\text { on WHO staging or CD4 }\end{array}$ & $4.4 \pm 1.1$ & $4.0 \pm 1.6$ & $-9 \%$ \\
\hline $\begin{array}{l}\text { Assessment of WHO clinical } \\
\text { staging at every visit }\end{array}$ & $4.6 \pm 0.7$ & $4.5 \pm 0.8$ & $-2 \%$ \\
\hline Screening for TB at each visit & $4.0 \pm 1.4$ & $4.6 \pm 1.0$ & $15 \%$ \\
\hline Monitoring for drug toxicity & $4.4 \pm 1.1$ & $4.4 \pm 0.9$ & $0 \%$ \\
\hline Assessment for adherence & $4.9 \pm 0.2$ & $4.9 \pm 0.5$ & $0 \%$ \\
\hline \multirow[t]{2}{*}{ Prescription of cotrimoxazole } & $4.8 \pm 0.4$ & $4.9 \pm 0.3$ & $2.1 \%$ \\
\hline & $4.1 \pm 0.8$ & $4.3 \pm 0.7$ & $4.9 \%$ \\
\hline \multicolumn{4}{|l|}{$\begin{array}{l}\text { Prescription of isoniazid for } \\
\text { those eligible }\end{array}$} \\
\hline $\begin{array}{l}\text { Request for CD4 count every } 6 \\
\text { months }\end{array}$ & 5.0 & 5.0 & $0 \%$ \\
\hline
\end{tabular}


Table 3 shows the percentage mean difference for each practice item. The mean difference for screening for TB at each visit was $15 \%$ which was the highest. The mean difference for prescription of cotrimoxazole was
2.1 while that for prescription of Isoniazid was $4.9 \%$. However, there was a negative mean difference for assessment of WHO staging at every visit $(-2 \%)$ and commencement of ART based on WHO staging or CD4 (-9\%).

Table 4: Grading of health workers knowledge of the HIV guideline

\begin{tabular}{lll}
\hline Knowledge level & $\begin{array}{l}\text { Pre- } \\
\text { intervention } \\
\mathbf{n}=\mathbf{3 3}\end{array}$ & $\begin{array}{l}\text { Post- } \\
\text { intervention }\end{array}$ \\
& Freq(\%) & $\mathbf{n} 8$ \\
& Freq(\%) \\
\hline Good & $32(97.0)$ & $27(96.4)$ \\
Poor & $1(3.0)$ & $1(3.6)$ \\
\hline
\end{tabular}

Table 4 shows that majority of the respondents had good knowledge of the guideline

Table 5: Grading of health workers practice of the HIV guideline

\begin{tabular}{lll}
\hline Practice level & Before & After \\
& $\mathbf{n}=\mathbf{3 3}$ & $\mathbf{n}=\mathbf{2 8}$ \\
& Freq(\%) & Freq(\%) \\
\hline Good Practice & $33(100)$ & $28(100)$ \\
Poor practice & $0(0)$ & $0(0)$ \\
\hline
\end{tabular}

Table 5 shows that all the respondents had good practice of the guidelines.

\section{Result from patient treatment cards}

Data from patient treatment card showed that WHO staging, screening for tuberculosis, prescription of cotrimoxazole, and assessment of adherence were being done at every visit for all the cards evaluated 6 months before the intervention. Also, baseline investigation and adherence counseling were done before commencement of antiretroviral therapy for all the treatment cards reviewed. CD4 count was being requested every 6 months for $52 \%$ of the reviewed cards and every 12 months for $48 \%$ of the reviewed cards.

\section{Result of Key informant interview}

The respondents affirmed that the guidelines are being implemented to a large extent, although one of the respondents pointed out that the test and treat guideline is not yet fully implemented. Below is a supporting quote: "The guideline is being implemented to a large extent. HIV Testing Services (HTS) are implemented almost fully except that HTS for sexual partner of positive individuals though emphasized is not optimally implemented"' (Community Physician 1)

The respondents reported that the health workers particularly the medical doctors working in the clinic have access to the guidelines. They also affirmed that the training is being carried out quarterly, though it hasn't been constant due to some challenges.

One of the respondents said "To some extent, I would say yes. Doctors in the clinic have access to the material but not all the other health workers, most of who base the management of patients on training they were given by partners and coworkers"(Community Physician 1) 
Another reported thus "Training on HIV management guideline used to be on Monthly bases until the project period of partners and donors elapsed mid last year. Though new partners have announced their presence and willingness to carry on with sponsorship, they are yet to start" (Nurse)

Some of the challenges highlighted by respondents include breakdown of CD4 machines, viral load not routinely assessed making it difficult to assess patient's progress, unavailability of printed copies of the guidelines in the clinic for reference purposes, clients not having money to pay for other lab investigations, too many information given at same time, challenges with training due to strike and change of implementing partners, and frequent rotation of health workers.

\section{Discussion}

The study examined health worker knowledge and practice of HIV management guideline and also appraised the implementation of the HIV management guideline in the adult ART clinic of Alex-Ekwueme Federal University Teaching Hospital Abakaliki. About half of the respondents were in the 34-43 year age group. Majority of the health workers were medical doctors and more than half had worked in the clinic for 3-5 years. Working experience has a part to play in improving knowledge and practice.

At pre-intervention, knowledge level of respondents about the HIV guidelines was high with $97 \%$ of respondents having good knowledge of the guidelines with a mean score of 3.9 out of 5 and standard deviation of 0.5 . This high knowledge can be explained by the fact that majority of the respondents had access to and had read the guidelines. About half of the respondents had also been trained on the guideline at some point. This good knowledge corroborates the findings of a study carried out to assess knowledge, attitude and practice of human immunodeficiency virus infection post-exposure prophylaxis among resident doctors in a tertiary hospital in Benin City, Nigeria which showed that majority of the respondents had good knowledge. ${ }^{14}$ This similarity may be explained by the fact that the study focused on post-exposure prophylaxis aspect of the guidelines which is an aspect that most health workers pay attention to due to fear of being infected in the process of caring for patients. This high level of knowledge also agrees with the result of a study in South Africa that determined the knowledge and practice of the prevention of mother-to-child transmission of HIV guidelines amongst doctors and nurses that revealed that respondents had high levels of knowledge (94\%) regarding HIV counselling and testing (HCT). ${ }^{9}$
This study also revealed that all the respondents had good practice of the guidelines at pre-intervention. The total mean score for practice was 4.5 out 5 and a standard deviation of 0.5 . This shows that healthcare providers who participated in this study had very good practice of HIV guideline which includes activities such as: Assessment of WHO clinical staging at every visit, screening for TB at each visit, monitoring for drug toxicity, requesting for CD4 count every six months, assessment for adherence, prescription of cotrimoxazole, and prescription of isoniazid for those eligible. This good practice may be because majority of the respondents were medical doctors who are mostly responsible for patient assessment and drug prescription. This finding is similar to the result of previous studies. ${ }^{15,16}$

The good practice reported by respondents agrees with the result from patient treatment card except for the request for $\mathrm{CD} 4$ count which showed that $\mathrm{CD} 4$ count was being requested every 6 months for $52 \%$ of the reviewed cards and every 12 months for $48 \%$ of the reviewed cards. These disparities may be attributed to the frequent breakdown of the CD4 cell count machine experienced by the health facility. Similarly, report from the key informants revealed that the guideline is being well implemented.

The intervention in this study was useful in improving the knowledge and practice of the HIV guidelines. The intervention involved provision of pamphlets for the health workers which contained summarized information from the guidelines. Educational interventions such as use of leaflets and pamphlets have been shown to improve knowledge and practice of health program implementation. ${ }^{17}$ The pamphlet that was used in this study provided a summary of the guidelines which made it easier for health workers to pick necessary information at a glance. It also served as a reference for the health workers especially during the clinics and was helpful in saving time since it's a summary of the main guidelines.

The improved knowledge agrees with the result of a study in Nepal on the effectiveness of educational intervention in improving knowledge and practice of universal precautions among nurses that revealed that there was significant difference in the pre and post-intervention test mean knowledge. ${ }^{18}$ However, in this present study, the mean knowledge score for commencement of ART regardless of WHO staging or CD4 count reduced from 4.0 to 3.8 with a percentage mean difference of minus 5\%. This decrease may be explained by the fact that at the time of the study, there was a transition from the 2014 guidelines to the 2016 guide- 
lines and the criteria for commencement of ART is one of the criteria that changed in the new guidelines. The old guidelines require that ART should be commenced based on WHO staging or CD4 count. But in the new guidelines, ART should be commenced regardless of WHO staging or CD4 count . ${ }^{7}$ The health workers may not have been informed about some of the changes in the guidelines and this may have affected their responses.

Mean scores for health worker practice also improved after the intervention. Although there was no increase in the overall mean practice score of 4.5.The mean score for some practice items increased with screening for TB at each visit having the highest increase from 4.0 to 4.6 giving a percentage mean increase of $15 \%$. Mean practice score for prescription of cotrimoxazole increased from 4.8 to 4.9 (percentage mean increase of $2.1 \%$ ) while that for prescription of isoniazid for those eligible increased from 4.1 to 4.3 (percentage mean increase of $4.9 \%$ ). This finding agrees with a study in Nepal which was carried out to determine the effectiveness of educational intervention in improving knowledge and practice of universal precautions among nurses and revealed that there was significant difference in the pre and post-intervention test mean practice. ${ }^{18}$ However, there was a decrease in the mean scores for commencement of ART based on WHO staging or CD4 with a mean difference of minus $9 \%$. This decrease in the mean score may also be as a result of the transition from the old guidelines to the new ones during the study period.

\section{Study limitations}

One limitation of this study is that it was carried out among health workers in one tertiary health facility. This will affect the extent to which the findings can be generalized. Additionally, this study did not include a control arm and so it is difficult to conclude that the intervention led to an improvement without having a comparison group. Also, previous trainings or other job aids could have been source of knowledge and could have influenced practice.

\section{Conclusion}

The study concludes that the HIV guidelines are being implemented in the clinic to a large extent and an educational intervention may be useful in improving and sustaining implementation. However there are some barriers to implementation. Trainings, more funding and provision of the guideline in the clinics will help in sustaining its implementation in the clinic. Policy makers should aim at producing summarized form of guidelines in addition to the main guideline for easy reference by health workers to promote effective implementation.

\section{Acknowledgement}

The authors wish to thank the respondents that participated in this study. We also wish to thank the African institute for health policy and health systems, Ebonyi State University, Nigeria for their support.

\section{Conflict of interest}

The authors declare that they have no conflict of interest

\section{References}

1. Rhodes RL, Nazir F, Lopez S, Xuan L. Use and Predictors of End-of-Life Care Among HIV Patients in a Safety Net Health System. J Pain Symptom Manage [Internet]. 2016;51(1):120-5. Available from: http://dx.doi. org/10.1016/j.jpainsymman.2015.08.010

2. Burua A, Nuwaha F, Waiswa P. Adherence to standards of quality HIV / AIDS care and antiretroviral therapy in the West Nile Region of Uganda. 2014;1-7. 3. Mwangome MN, Geubbels E, Wringe A, Todd J, Klatser P, Dieleman M. A qualitative study of the determinants of HIV guidelines implementation in two South-Eastern districts of Tanzania. 2017; (March):825-34.

4. Mazrou SHA. Expected benefits of clinical practice guidelines: Factors affecting their adherence and methods of implementation and dissemination. 2013;1(3).

5. Federal Ministry of Health. Integrated national guidelines for HIV prevention, treatment, and care: Chapters 5 and 6. 2015;47-55. World Health Organisation. Policy Brief Consolidated guidelines on the use of antiretroviral drugs for treating and preventing HIV infection: What's new. 2015; (November).

7. Federal Ministry of Health. National guidelines for HIV prevention, treatment and care. National AIDS and STI's control programme. 2016.

8. Evenblij K, Verbon A, Leth F Van. Intention of physicians to implement guidelines for screening and treatment of latent tuberculosis infection in HIV-infected patients in The Netherlands: a mixed- method design. BMC Public Health. 2016;1-9. Available from: http:/ /dx.doi.org/10.1186/s12889-016-3539-2

9. Ogbonna K, Govender I, Tumbo J. Knowledge and practice of the prevention of mother-to-child transmission of HIV guidelines amongst doctors and nurses at Odi Hospital , Tshwane District Knowledge and prac- 
tice of the prevention of mother-to-child transmission of HIV guidelines amongst doc. South African Fam Pract. 2016;58(5):167-71. Available from: http://dx.doi.org/ 10.1080/20786190.2016.1228561

10. Pakenham-Walsh N, Bukachi F. Information needs of health care workers in developing countries: a literature review with a focus on Africa. Hum Resour Health. 2009;7(1):30. Available from: http://www.ncbi.nlm.nih. gov/pubmed/19356239

11. Mathewos B, Birhan W, Kinfe S, Boru M, Tiruneh G, Addis Z, et al. Assessment of knowledge, attitude and practice towards post exposure prophylaxis for HIV among health care workers in Gondar, North West Ethiopia. BMC Public Health. 2013 May 25;13:508. Available from: http://www.ncbi.nlm.nih.gov/pubmed/23705668

12. Giese S, Hussey G. Rapid appraisal of primary level health care services for HIV-positive children at public sector clinics in South Africa. 2002.

13. Quach L, Mayer K, McGarvey ST, Lurie MN, Do P. Knowledge, attitudes, and practices among physicians on HIV/AIDS in Quang Ninh, Vietnam. AIDS Patient Care STDS. 2005 May;19(5):335-46. Available from: http://www.ncbi.nlm.nih.gov/pubmed/15916496

14. Ekundayo OJ, Aliyu MH, Jolly PE. A Review of Intestinal Helminthiasis in Nigeria and the need for school-based intervention. I Rural Trop Public Heal. 2007;6:33-9 PubMed .

15. Uzodike N, Ross A, Harbor O. Adherence by a primary healthcare clinic in KwaZulu-Natal to the national HIV guidelines Adherence by a primary healthcare clinic in KwaZulu-Natal to the national HIV guidelines. South African Fam Pract. 2015;57(3):198-202. Available from: http://dx.doi.org/10.1080/20786190.2014.9769 45

16. Dasgupta ANZ, Wringe A, Crampin AC, Chisambo C, Koole O. HIV policy and implementation: a national policy review and an implementation case study of a rural area of northern Malawi †. AIDS Care. 2016;28(9):1097 PubMed -109.

17. Cooper CM, Ahmed S, Winch PJ, Pfitzer A, Mckaig $\mathrm{C}$, Baqui AH. Patient Education and Counseling Findings from the use of a narrative story and leaflet to influence shifts along the behavior change continuum toward postpartum contraceptive uptake in Sylhet District , Bangladesh. Patient Educ Couns. 2014;97(3):37682. Available from: http://dx.doi.org/10.1016/j. pec.2014.09.007

18. Indra M, Rai S. Indra Shrestha : Educational Intervention on Universal Precautions Among Nurses Impact of Educational Intervention on Knowledge and Practice of Universal Precautions among Nurses. 2014;1(1):57-60. 